\title{
SONIFICATION OF MOVEMENT FOR MOTOR SKILL LEARNING IN A NOVEL BIMANUAL TASK: AESTHETICS AND RETENTION STRATEGIES
}

\author{
John Dyer ${ }^{1}$, Paul Stapleton ${ }^{2} \&$ Matthew Rodger ${ }^{1}$ \\ School of Psychology ${ }^{1} \&$ Sonic Arts Research Centre ${ }^{2}$, \\ Queen's University Belfast, \\ University Road, \\ BT7 1NN \\ jdyer01@qub.ac.uk
}

\begin{abstract}
Here we report early results from an experiment designed to investigate the use of sonification for the learning of a novel perceptual-motor skill. We find that sonification which employs melody is more effective than a strategy which provides only bare timing information. We additionally show that it might be possible to 'refresh' learning after performance has waned following training - through passive listening to the sound that would be produced by perfect performance. Implications of these findings are discussed in terms of general motor performance enhancement and sonic feedback design.
\end{abstract}

\section{INTRODUCTION}

Sonification of human movement is slowly becoming a more commonly-used strategy for the provision of augmented perceptual feedback for motor skill learning [1]-[4]. Typically, this entails some variable of motor performance being tracked by a sensing system (e.g. accelerometers, optical motion capture, touchpads, force-plates) and fed back 'live' to the moving individual in the form of synthesized sound [5]. Making movement information available through sound where it would otherwise be difficult to perceive can allow a learner to exert much finer control over their actions, ideally resulting in better task performance [6]. In this report, we aim to explore the benefits of making sonification musical (as compared to sonification concerned purely with providing temporal sonic information), and test a strategy for improving long-term retention of new skills learned with sonification.

\section{THE VALUE OF SONIFICATION FOR PERCEPTUAL-MOTOR LEARNING}

In the psychology of motor skill learning and feedback, a major concern is the transfer of learned motor skills beyond the feedback environment [7]. Traditionally, the consensus has been that overuse of augmented feedback leads to dependence, as learners come to over-rely on the guidance it provides [8]. This "guidance effect" has been assumed to apply to all types of augmented feedback, independent of sensory modality or form, however this assumption is based

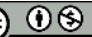

(c) This work is licensed under Creative Commons Attribution - Non Commercial 4.0 International License.

The full terms of the License are available at

http://creativecommons.org/licenses/by-nc/4.0/ almost entirely on research employing transformed or abstracted visual feedback. When sonification has been compared directly to such feedback, the guidance effect fails to materialize; task performance remains at a high level [9]. Retention tests without live sonification are crucial in this domain, as the goal is to learn an underlying movement skill; sonification is the vehicle for getting there more quickly, or learning the skill more accurately. Both everyday and sportrelated skills (the usual targets of this treatment) should ideally not be dependent on immersion within a feedback system. Findings such as the above suggest that sonification could in fact be a more appropriate type of augmented feedback for skill learning than the same information provided via a visual display (for more on this idea, see [10]).

\section{AESTHETIC ISSUES}

Where the efficacy of sonification has been tested experimentally, feedback systems have sometimes made use of aesthetically impoverished movement-sound mapping strategies. Pitch-mapping is the most common strategy in sonification generally [11], and the same can be said for the more narrow subdomain of sonification for perceptual-motor feedback. Konttinen et al., [12] for example, mapped deviation from a target to sine-tone pitch in a shooting task to provide feedback for use in controlling rifle stability. Schaffert and Mattes [13] mapped boat acceleration to the pitch of discrete tones in a MIDI note scale, while Powell and Lumsden [14] employed tone pitch to allow drivers to perceive their lateral g-force relative to a set limit in a motorsport racing task. More complex and interesting sounds have also been used to provide information about human movement, including vowel-like sounds (in golf swinging and jumping [5], [15]) and physical modelling of real-world noisy interactions (in handwriting [1]). Direct comparisons of basic vs. pleasant (but structurally similar) mapping strategies for motor skill learning have rarely been explored experimentally.

For a novel motor task which has not been sonified before, it can be difficult to know the extent to which one should focus on aesthetics when designing sound as feedback. Simple approaches to sonification which provide basic temporal information to help organize performance have been shown to be effective for learning new tasks [3], [9], however there could yet be potential benefits unlocked through use of a more interesting mapping. Motivational factors are seldom considered in perceptual-motor learning studies, despite their importance for task engagement and therefore, performance [16]. Sonification as feedback presents a unique opportunity to provide augmented perceptual information which is 
pleasant, evocative and interesting to use; to carry over information-design habits from classic visual feedback experiments (which have historically provided performance information as moving lines on a screen e.g. [17], [18]) might be a tremendous waste of potential. This is one of the dilemmas we aim to probe with the current investigation - by comparing one type of sonification which provides only temporal information, to another which also employs melody.

\section{PROLONGING RETENTION}

As mentioned in section 2, good performance after the removal of sonified feedback is the goal of our research. It is not always practical to provide a live auditory display during motor performance, thus we require learning which is not dependent on the immediate presence of augmented feedback, i.e. learning that generalizes - and is not subject to the 'guidance effect' [8]. In a previous study by the authors similar to the current investigation (manuscript under revision at time of writing), we observed better performance in a custom bimanual shape-tracing task by participants learning under sonification conditions relative to control (i.e. silence). This task requires participants to trace a triangle with the left index finger while simultaneously tracing a diamond with the right (see Figure 1). When the task is performed correctly, participants move between corner zones with a timing ratio of $4: 3$. This type of bimanual dual-task is difficult to perform, but can be learned quickly with the use of augmented perceptual feedback [17]. Such feedback works by integrating perception of both hands into a single perceptual stream, which is more easily controllable [19]. When we removed sonification to test retention-withoutfeedback, the boosted performance by participants in that group remained; there was no evidence of a 'guidance effect'. However, in a second retention-without-feedback session 24 hours later, performance had declined and there was no longer a difference in scores between sonification and control.

\subsection{Listening for retention}

It may be possible to temporally extend the advantage of sonification by allowing participants to hear the sound of good performance before no-feedback retention-testing. It has been shown in musical instrument learning that listening to a learned piece of music elicits activations in neural areas associated with performing the piece [20]. It has been argued that this and similar such findings represent a mechanism of 'common coding' for perception and action in the brain [21]. In other words, perceptual experience of learned action is neurally very similar to active performance. This could be exploited to enhance recall of new motor skills in sonification, as has been demonstrated in keyboard learning [22]. If this strategy works, it could have implications for how sonification-based training should be implemented in real-life skills. Playing a recording is much less onerous than providing live sonification. For example, a sporting skill, say, a golf swing [15] can be trained using sonification in a lab setting. Sonification might enhance performance of the swing in the lab by making temporal information about bodily rotation more perceivable, and the learner may come to understand their action in terms of its sonic outcome. Through practice, it is expected that the learner would come to know the sound of a good swing and purposely act so as to produce it. On the golf course, where it may be impractical to use live sonification (perhaps due to cumbersome equipment), the learner could listen to the sound of a good swing through headphones, and thus re-experience (part of) what it is like to produce a good swing, thereby enhancing motor sequence retention.

In the current experiment, we test this strategy by reexposing participants to the sound of good performance prior to a 24-hour retention test, with the expectation that doing so should improve performance.

\section{METHOD}

Participants were recruited from the university undergraduate population (currently $\mathrm{N}=45$ ) and randomly allocated to one of three independent conditions.

All were required to learn the same bimanual shape-tracing task (Figure 1). Participants were instructed (via an animated demonstration) to trace two shapes (a triangle and a diamond) in an anticlockwise direction starting from the top corners, and to arrive at corner zones at regular intervals on each hand. When done correctly, the fingertips of both hands would complete a cycle (i.e. return to the top corner) at the same time. Task performance required continuous repeated cycles of the shapes.

Movements were tracked using reflective markers attached to a pair of modified golfing gloves which were picked up by four optical motion-capture cameras. Sonification was provided (where necessary) by streaming Cartesian coordinate data corresponding to the position of the fingertip marker of each hand into Max/MSP 6.0 at $20 \mathrm{~Hz}$.

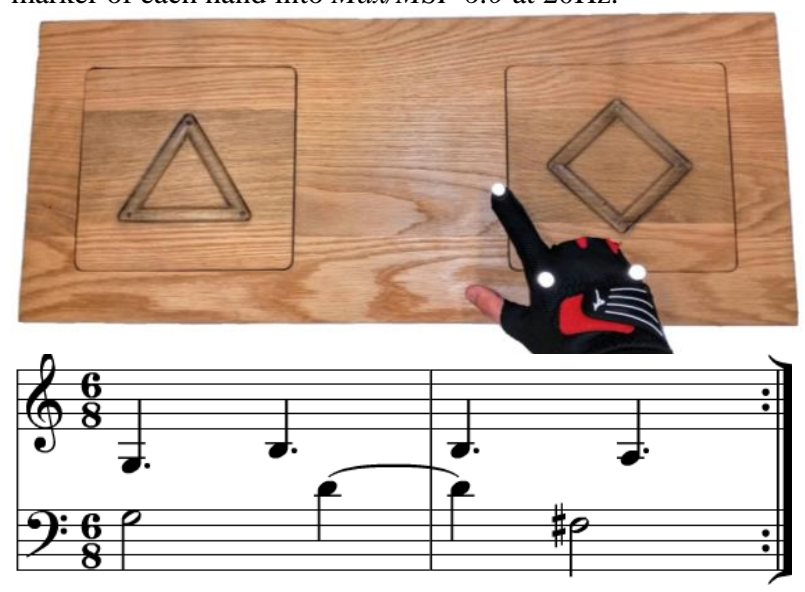

Figure 1: Custom bimanual shape-tracing apparatus used in the reported experiment (top) and notes produced by the sonification patch in the 'Melodic' experimental condition (bottom). This melody was composed by the authors for the purpose of the experiment.

One group of participants $(\mathrm{N}=15)$ was required to learn the task without sonification of any kind (the 'Control' condition). This group listened to pink noise through headphones during practice. Another group $(\mathrm{N}=15)$ practiced with basic sonification of fingertip corner arrivals (the 'Temporal' sonification condition). When a fingertip reached a corner, a short $(200 \mathrm{~ms})$ burst of white noise was triggered. Correct performance thus produced a 4:3 rhythm. A third group $(\mathrm{N}=15)$ practiced with melodic sonification of fingertip arrivals (the 'Melodic' sonification condition). In this condition, correct performance of the task produced a simple melody (right - left hand notes occurring in a $4: 3$ rhythm) on a synthesized plucked stringed instrument in the 
key of G major (see Figure 1). Sounds were presented through headphones.

The procedure of this experiment consisted of three main stages: pretest, practice and retention testing.

\subsection{Pretest}

Participants completed a pretest trial at the beginning of the session. A demo animation was played to participants prior to the pretest which showed corner arrivals occurring as they would if the task were performed perfectly. The demo lasted 9 seconds and consisted of three cycles of the shapes. For the pretest, all participants heard constant pink noise to obscure potential task-intrinsic auditory feedback. The movement phase consisted of a 26-second window in which participants attempted to match the demonstration. No artificial feedback was provided.

\subsection{Practice}

Following the pretest, participants underwent fourteen, 26second-long practice trials - the nature of which varied depending on condition assignment. The demo animation was played prior to every practice trial. Participants in the Control condition heard pink noise during demo presentation and the movement phase. Participants in the Temporal sonification condition heard $200 \mathrm{~ms}$ bursts of white noise coincident with corner arrivals while the demo played and subsequently with their own corner arrivals on the shapes. Participants in the Melodic sonification condition heard the notes shown in Figure 1 coincident with corner arrivals in the demo and their own on the shapes. Participants did not commence movement until the demo had concluded. For participants in the sonification conditions, engaging in this task was thus instantiated as an unfolding sonic performance, and practice trials as repeated attempts to 'play' the task correctly. Participants in all groups received terminal (posttrial) feedback in the form of their inter-manual timing ratio plotted over time.

\subsection{Retention testing}

Following the practice phase, all participants immediately underwent a retention test with no demo, terminal (graph) feedback or sound except for constant pink noise during the movement phase.

Another retention test under exactly the same conditions was administered the following day.

Participants in the two sonification conditions were then reexposed to the sound of perfect task performance. Note, they did not see the demo animation again, only the sound it produced during the practice phase the day before. Participants then completed another retention test. To control for potential practice effects of multiple-retention tests, participants in the control condition also completed this additional retention test, but did not hear any sound other than constant pink noise. Participants also completed a questionnaire asking about their experience of the experiment (enjoyment, interest and strategies used) and musical experience.

\section{RESULTS AND DISCUSSION}

The main measure of performance in the current task is the bimanual timing ratio produced by participants over time.
Within each trial, the absolute difference between produced and ideal (4:3) timing ratios was averaged to produce a single error score for each trial for each participant. As learner performance after practice is our primary interest, we here present a preliminary analysis of data from trial 14 and the following three retention tests (i.e. the final four stages shown in Figure 2 for all 3 conditions and all 45 current participants). A mixed ANOVA with trial and feedback group as factors revealed a significant main effect of feedback group: $F(2,39)=3.579, p=0.037$, no significant main effect of trial: $F(2.147,83.744)=2.593, p=0.077$ and no significant interaction: $F(4.295,39)=0.572, p=0.696$. Our analysis does not currently go further because we are still in the process of collecting data, with the aim of $\mathrm{N}=60$. Average absolute bimanual ratio error over pretest, practice and retention

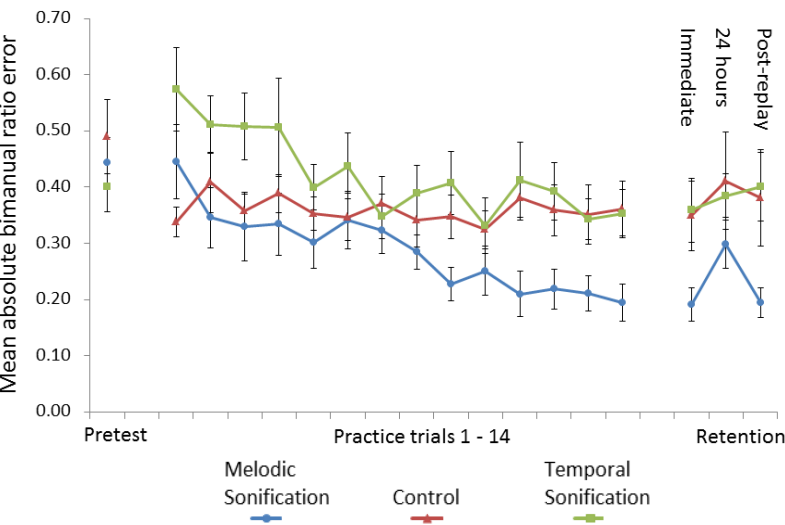

Figure 2: Rates of error for the three experimental groups over time. Live sonification and terminal feedback were provided only on practice trials. The three retention means show no-feedback error rates following practice, after 24 hours, and lastly, after listening to the sound of perfect performance. Error bars are standard error of the mean.

Figure 2 shows different rates of reduction in error for experimental groups over time. Participants in the Melodic sonification condition reached lower average error scores than the Temporal sonification and Control conditions. This indicates that Melodic sonification was most useful for acquisition of the bimanual skill. The same pattern is observed at the first retention test, in which no sonification feedback was available. This indicates that participants in the Melodic sonification condition were not dependent on the presence of augmented feedback for good performance. On the second retention test (after 24 hours), average ratio error in the Melodic sonification condition increases to levels similar to the Temporal sonification and Control conditions. However, subsequently re-exposing participants to the sound of good performance appears to have had the desired effect, at least in the Melodic condition - error reduces in line with performance on the previous day. No benefit of hearing the sound of good performance seems evident in the Temporal condition, and there is little if any practice effect for the Control condition on day 2.

Limited conclusions can be drawn from this incomplete dataset and the preliminary analysis we have conducted here. The lack of an enhancement effect of sonification in the Temporal condition is surprising, but may be related to motivation (the sound is very dull), or informationalstructural factors (the melody specifies the ordering of bimanual movements, making the task relatively easier in that condition). This may become clear with further analysis including questionnaire data. 


\section{CONCLUSION}

We have presented preliminary evidence which indicates potential for improving motor task retention with passive listening. This means that if a new motor skill is learned with sonification, it may be possible to effectively 'refresh' learning through listening, rather than placing learners back in an augmented feedback environment.

The value of melody and engaging sound vs. purely temporal sonic information for learning in this task may be partly motivational, but could perhaps be related to the extra, relevant information provided by the use of different tones. Feedback designers should consider using melodic movement sonification for either or both of these reasons.

\section{REFERENCES}

[1] J. Danna, M. Fontaine, V. Paz-Villagrán, C. Gondre, E. Thoret, M. Aramaki, R. Kronland-Martinet, S. Ystad, and J.-L. Velay, "The effect of real-time auditory feedback on learning new characters.," Hum. Mov. Sci., vol. 43, pp. 216-228, Dec. 2015.

[2] R. Sigrist, G. Rauter, L. Marchal-Crespo, R. Riener, and P. Wolf, "Sonification and haptic feedback in addition to visual feedback enhances complex motor task learning.," Exp. brain Res., vol. 233, pp. 909925, Dec. 2014.

[3] F. T. van Vugt and B. Tillmann, "Auditory feedback in error-based learning of motor regularity.," Brain Res., vol. 1606, pp. 54-67, May 2015.

[4] F. Sors, M. Murgia, I. Santoro, and T. Agostini, "Audio-Based Interventions in Sport," Open Psychol. J., vol. 8, no. 3, pp. 212-219, 2015.

[5] A. O. Effenberg, "Movement Sonification: Effects on Perception and Action," IEEE Multimed., vol. 12, no. 2, pp. 53-59, Apr. 2005.

[6] J. Stienstra, K. Overbeeke, and S. Wensveen, "Embodying complexity through movement sonification," in Proceedings of the 9th ACM SIGCHI Italian Chapter International Conference on Computer-Human Interaction: Facing Complexity, 2011, pp. 39-44.

[7] R. Sigrist, G. Rauter, R. Riener, and P. Wolf, "Augmented visual, auditory, haptic, and multimodal feedback in motor learning: a review.," Psychon. Bull. Rev., vol. 20, no. 1, pp. 21-53, Feb. 2013.

[8] D. I. Anderson, R. A. Magill, H. Sekiya, and G. Ryan, "Support for an explanation of the guidance effect in motor skill learning.," J. Mot. Behav., vol. 37, no. 3, pp. 231-8, May 2005.

[9] R. Ronsse, V. Puttemans, J. P. Coxon, D. J. Goble, J. Wagemans, N. Wenderoth, and S. P. Swinnen, "Motor learning with augmented feedback: modalitydependent behavioral and neural consequences.," Cereb. Cortex, vol. 21, no. 6, pp. 1283-94, Jun. 2011.

[10] J. Dyer, P. Stapleton, and M. W. Rodger, "Sonification as concurrent augmented feedback for motor skill learning and the importance of mapping design," Open Psychol. J., vol. 8, no. 3, pp. 1-11, 2015.

[11] G. Dubus and R. Bresin, "A systematic review of mapping strategies for the sonification of physical quantities.," PLoS One, vol. 8, no. 12, p. e82491,
Jan. 2013.

[12] N. Konttinen, K. Mononen, J. T. Viitasalo, and T. Mets, "The effects of augmented auditory feedback on psychomotor skill learning in precision shooting," J. Sport Exerc. Psychol., vol. 26, pp. 306-316, 2004.

[13] N. Schaffert and K. Mattes, "Effects of acoustic feedback training in elite-standard Para-Rowing.," $J$. Sports Sci., vol. 33, no. 4, pp. 411-8, Jan. 2015.

[14] N. Powell and J. Lumsden, "Exploring novel auditory displays for supporting accelerated skills acquisition and enhanced performance in motorsport," in Proceedings of The 21st International Conference on Auditory Display (ICAD-2015), 2015, pp. 173-180.

[15] M. Kleiman-Weiner and J. Berger, "The sound of one arm swinging: A model for multidimensional auditory display of physical motion," in 12th International Conference on Auditory Display (ICAD), London, UK, June 20-23, 2006.

[16] G. Wulf, C. H. Shea, and R. Lewthwaite, "Motor skill learning and performance: a review of influential factors.," Med. Educ., vol. 44, no. 1, pp. 75-84, Jan. 2010.

[17] A. J. Kovacs and C. H. Shea, "The learning of $90^{\circ}$ continuous relative phase with and without Lissajous feedback: external and internally generated bimanual coordination.," Acta Psychol. (Amst)., vol. 136, no. 3, pp. 311-20, Mar. 2011.

[18] D. W. Vander Linden, J. H. Cauraugh, and T. A. Greene, "The effect of frequency of kinetic feedback on learning an isometric force production task in nondisabled subjects.," Phys. Ther., vol. 73, no. 2, pp. 79-87, Feb. 1993.

[19] E. A. Franz and R. McCormick, "Conceptual unifying constraints override sensorimotor interference during anticipatory control of bimanual actions.," Exp. brain Res., vol. 205, no. 2, pp. 27382, Aug. 2010.

[20] A. Lahav, E. Saltzman, and G. Schlaug, "Action representation of sound: audiomotor recognition network while listening to newly acquired actions.," J. Neurosci., vol. 27, no. 2, pp. 308-14, Jan. 2007.

[21] W. Prinz, "Perception and Action Planning," Eur. J. Cogn. Psychol., vol. 9, no. 2, pp. 129-154, Sep. 2010.

[22] A. Lahav, T. Katz, R. Chess, and E. Saltzman, "Improved motor sequence retention by motionless listening.," Psychol. Res., vol. 77, no. 3, pp. 310-9, May 2013. 